\title{
An Unusual Differential Diagnosis of Neck Swelling: Parathyroid Cyst
}

\author{
Kanu Kapoor, Imad Ali, Rajesh Agarwal, T.K. Thusoo, Anshuman Kaushal \\ Department of General Surgery, Artemis Hospital, Gurugram, Haryana, India.
}

\section{Corresponding Author: \\ Dr Kanu Kapoor \\ Email: kanu.kapoor21@gmail.com \\ This is an Open Access article distributed under the terms of the Creative Commons Attribution License (creativecommons.org/ licenses/by/3.0).}

Received

\begin{abstract}
Background: Differential diagnosis of cystic mass in the neck include thyroid cyst, thymic cyst, thyroglossal duct cyst, branchial cleft cyst, bronchogenic cyst, lymphangioma and parathyroid cyst (PC). Parathyroid cysts are rare and account for only $0.5 \%$ of all parathyroid pathologies. Case Report: A 30-year-old female with insidious onset anterior neck cystic swelling was diagnosed as benign parathyroid mass. Conclusion: Parathyroid cysts should be taken into consideration while evaluating asymptomatic neck masses.
\end{abstract}

Keywords: Biopsy, Neck, Parathyroid Diseases, Parathyroid Glands, Parathyroid Hormone.

\section{Introduction}

Parathyroid cysts (PC) represent $1-5 \%$ of all the neck masses [1]. The first description of parathyroid cyst was given in 1880 by Swedish anatomist Sandstrom [2]. Common sites for detection of PC cysts are anterior neck and mediastinum. More than 350 cases have been reported worldwide [3]. These cysts can be functional or non-functional depending on the presence or absence of symptoms of hyper-parathyroidism and treatment. Diagnosis of PC is made by monitoring serum calcium levels and cyst fluid analysis for parathyroid hormone. We hereby present the case report of a patient who presented to us with a painful cystic swelling in the anterior neck.

\section{Case Report}

A 30-year-old lady presented with the complaints of a left sided anterior neck mass [Fig.1] for 3 months. This was insidious in onset and gradually increasing in size, associated with tenderness since the past one month. She did not report any disturbance in swallowing or changes in voice or dyspnea. On examination a small cystic swelling was palpated in the anterior triangle of the neck behind the sternal head of the left sternocleidomastoid, which was tender to touch, fluctuant, showed no movement with deglutition and the skin overlying the swelling was normal, rest of the neck examination was found to be within normal limits. MRI revealed $4 \times 3 \times 2.5 \mathrm{~cm}$ sized thin-walled cystic lesion in the lower part of left anterior triangle of neck, deep to lower part of the left sternocleidomastoid muscle. The cyst abuts the trachea on the right side and the left common

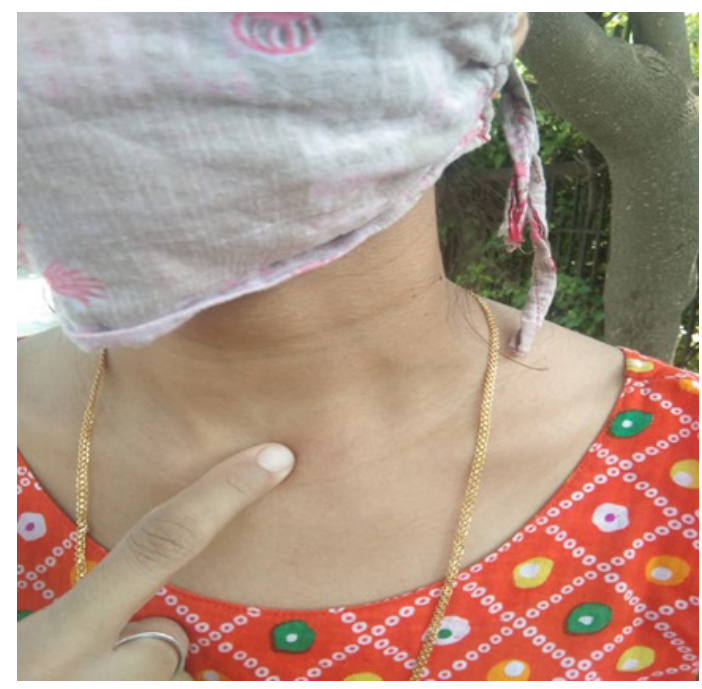

Fig.1: Mass in the left anterior triangle of the neck. 
carotid artery on the left side with preserved intervening fat planes. The cyst abuts the inferior surface of the left lobe of the thyroid reaching upto the parathyroid region. However fat planes with the left lobe of the thyroid appear predominantly intact [Fig.2].

The patient was taken up for excision biopsy of the cyst and intra-operatively the swelling was present deep to the sternal head of the left sternocleidomastoid muscle and the strap muscles had to be dissected to gain access to it, in order to excise it completely. The cyst was filled with clear fluid was excised in toto and sent for histopathological evaluation [Fig.3]. Microscopic evaluation showed a fibrous cyst wall line by flattened parathyroid chief cells and clear cells with small islands of parathyroid tissue within the wall along with focal adjacent unremarkable parathyroid tissue suggestive of a benign parathyroid cyst [Fig.4]. Her serum intact parathyroid hormone levels were found to be $19.50 \mathrm{pg} / \mathrm{mL}$ (normal 14$72 \mathrm{pg} / \mathrm{mL}$ ) and serum calcium was found to be 9.09 $\mathrm{mg} / \mathrm{dL}$ (normal 8.80-10.60 mg/dL). Patient was followed up a week after surgery and wound was found to be healthy [Fig.5].

\section{Discussion}

PCs present as solitary orthotropic, non-functioning symptomatic neck swellings in the region of inferior pole of the thyroid in $80 \%$ of the cases. These most commonly present as neck swellings but can also present as mediastinal masses. PCs can present as functioning or non-functioning cysts. Nonfunctioning cysts are a more common presentation, occurring more commonly in women (male: female ratio $1: 25)$, in the $4-5^{\text {th }}$ decade of life and $70 \%$ of these arise from the inferior parathyroid gland [5]. However, $10 \%$ of all PCs present as a functional cyst with features of hyperparathyroidism. These have a male preponderance and show elevated levels of C-terminal PTH (C-PTH) levels [6].

Pathogenesis of PCs remains unclear, but several theories have been proposed for the
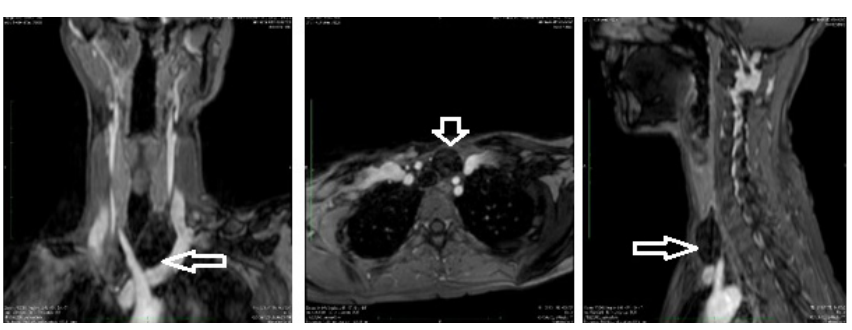

Fig.2: MRI images with white arrow mark pointing towards the cyst.

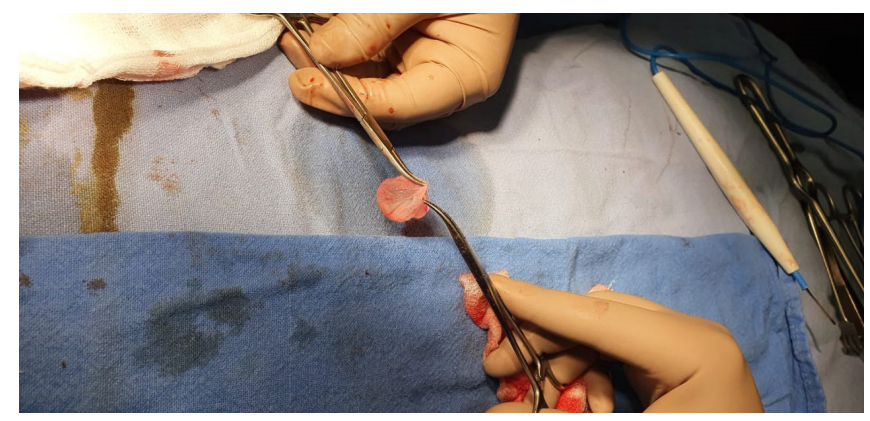

Fig.3: Intra-operative images showing cystic swelling present deep in the anterior triangle of the neck with second image showing cyst filled with clear fluid.

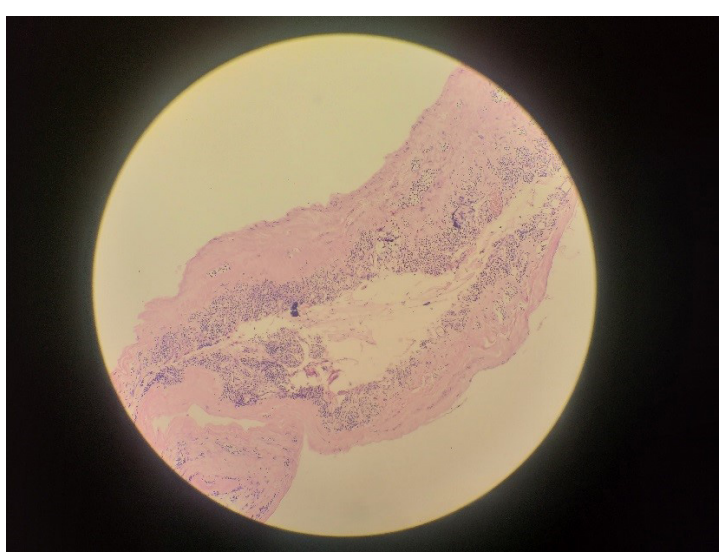

Fig.4: Histopathological image of the cyst wall.

same and it is proposed that these cysts result from a vestigial remnant of the third or fourth brachial cleft or from the persistence of Kürsteiner canals [8]. Other theories propose that PCs develop from the infarction and degeneration of a parathyroid adenoma or from the coalescence of multiple microcysts in normal parathyroid tissue [4]. Microcysts are a common finding in the ageing parathyroid glands and autopsy material 
reveals that up to $50 \%$ of asymptomatic glands contain microcysts [7]. Conversely, microcysts are uncommon and always warrant clinical investigation.

Evaluation of a PC involves a thorough head and neck examination followed by fine needle aspiration (FNA) and ultrasound (USG) imaging. Fine-needle aspiration often reveals a clear, colorless cystic fluid and pre-operative diagnosis of $\mathrm{PC}$ can be confirmed by demonstrating raised levels of C-PTH, irrespective of whether the cyst is functioning or non-functioning $[9,10]$. C-PTH is measured in cystic fluid rather than intact PTH levels as non-functioning parathyroid cysts produce large quantities of PTH which gets rapidly degraded into the biologically inactive C-terminal peptide [9]. Hence, parathyroid cysts may have a relatively low intact PTH level, underestimating their ability to produce PTH and resulting in a false negative diagnosis [9]. Even though imaging forms a very useful tool for the pre-operative assessment of such lesions, there are no specific radiological methods for differentiating parathyroid cysts from other cystic neck lesions as USG neck only reveals a non-specific cystic structure. CT and MRI neck not only help demonstrate the cystic component of these lesions but also help to visualize its relationship with adjacent tissues. These imaging modalities are particularly useful in the presence of substernal extension or in the presence of compressive symptoms. Radioiodine thyroid scanning has been used to detect a non-functioning cold nodule and helps differentiating a thyroid nodule from a parathyroid cyst. Despite the ability to detect PC pre-operatively, most parathyroid cysts are only diagnosed post-operatively.

Definitive treatment for PCs is cyst excision although USG guided FNA is a safe and easy technique used in the primary management and is both diagnostic and therapeutic. The only drawback of the procedure being recurrence. Multiple studies have evaluated the success rate of simple aspiration of $\mathrm{PC}$ alone, with therapeutic

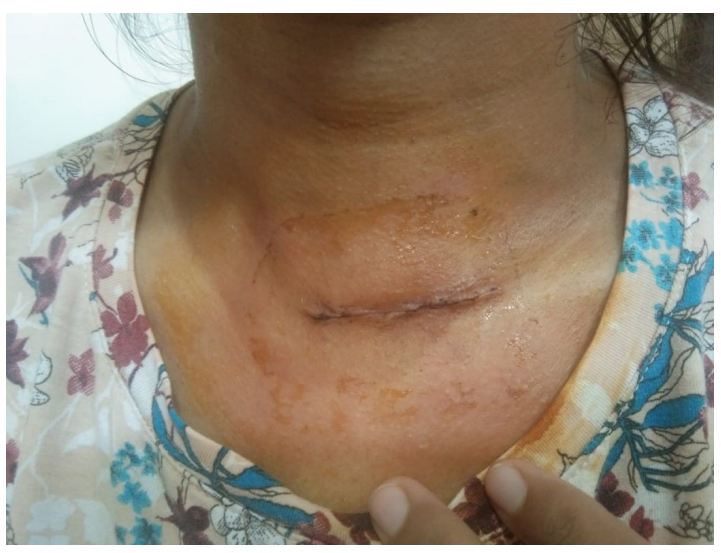

Fig.5: Post-operative image of the wound found to be healing well by primary intention.

success rates between $30-90 \%$ [6]. Sclerotherapy is another option and can also be used as a treatment modality with increased risk of complication like extra-cystic fibrosis and recurrent laryngeal nerve palsy and subsequent vocal cord palsy following the use of sclerosing agents with poor longterm efficacy and is hence not a widely accepted modality.

Microscopically, non-functioning PC have a smooth internal layer with a thin membranous layer. The cyst wall is formed by a single layer of cuboidal or columnar epithelium which stains positive for glycogen. Macroscopic features that help us to differentiate it from other lesions are a smooth, shiny, semi-transparent thin cyst wall that is usually loosely attached to the thyroid and is easily dissected free from it and the surrounding tissue. Intra-operative cyst rupture should be avoided in order to prevent parathyromatosis [11]. Postoperatively serum calcium levels are monitored as hypocalcemia may occur following parathyroid cyst excision. Patients with large cysts $(\geq 4 \mathrm{~g})$ are at a higher risk of symptomatic hypocalcemia and should be managed accordingly [11].

In our study the patient presented to us with an asymptomatic progressively enlarging neck mass which is the most common assumption, with compressive symptoms being the second most frequent clinical presentation. Patient was taken up 
for excision biopsy after radiological investigations and histopathological features were suggestive of PC.

\section{Conclusion}

Parathyroid cysts are one of the rare parathyroid pathologies and should be taken into consideration in case of evaluation of suspected parathyroid pathologies or while evaluating asymptomatic neck masses. USG along with FNA guided cyst fluid aspiration help in aiding the diagnosis. Definitive treatment includes complete surgical excision and histopathological and cytological evaluation of the cyst.

Contributors: AK conceived the concept. KK wrote the first draft of the manuscript. IA, RA and TK helped with the study design and discussion. KK will act as guarantor. All authors approved the final version of this manuscript and are responsible for all aspects of this study.

Funding: None; Competing interests: None stated.

\section{References}

1. Cappelli C, Rotondi M, Pirola I, De Martino E, Leporati $\mathrm{P}$, Magri F, et al. Prevalence of parathyroid cysts by neck ultrasound scan in unselected patients. Journal of Endocrinological Investigation. 2009;32:357-359.

2. Sandstrom I. Om enkortel hos mennishkanochatskilligadoggdjus. Ups LakaforForhandl, 1880;14:441-471.
3. Papavramidis TS, Chorti A, Pliakos I, Panidis S, Michalopoulos A. Parathyroid cysts. Medicine. 2018;97:e11399.

4. Pontikides N, Karras S, Kaprara A, Cheva A, Doumas A, Botsios D, et al. Diagnostic and therapeutic review of cystic parathyroid lesions. Hormones. 2012;11:410-418.

5. da Silva DA, Machado MC, Brito LL, Guimarães RA, Fava AS, Mamone MCAC. Parathyroid cysts: diagnosis and treatment. Revista Brasileira de Otorrinolaringologia. 2004;70:840-844.

6. Ippolito G, Palazzo FF, Sebag F, Sierra M, de Micco C, Henry JF. A single-institution 25-year review of true parathyroid cysts. Langenbeck's Archives of Surgery. 2006;391:13-18.

7. Fortson JK, Patel VG, Henderson VJ. Parathyroid cysts: a case report and review of the literature. Laryngoscope. 2001;111:1726-1728.

8. Rossi ED, Revelli L, Giustozzi E, Straccia P, Stigliano E, Lombardi CP, et al. Large non-functioning parathyroid cysts: our institutional experience of a rare entity and a possible pitfall in thyroid cytology. Cytopathology. 2015;26:114-121.

9. Lee SL. Parathyroid cyst fluid: discrepancy between C-terminal and intact parathyroid hormone assays. Thyroid. 2000;10:1125-1126.

10. Guner A, Karyagar S, Ozkan O, Kece C, Reis E. Parathyroid cyst: the forgotten diagnosis of a neck mass. Journal of Surgical Case Reports. 2011;8:1-4.

11. Pirundini P, Zarif A, Wihbey JG. A rare manifestation of parathyroid carcinoma presenting as a cystic neck mass. Connecticut Medicine. 1998;62:195-197. 